\title{
Da vinci SILS-appendectomy for appendiceal endometriosis secondary to stage IV endometriosis: a case report and review of literature
}

\begin{abstract}
Background: Endometriosis is defined as functional endometrial tissue located outside of the uterine cavity. Typical symptoms include dysmenorrhea, non-cyclic dyspareunia, irregular or heavy menstrual bleeding, dyschezia, and infertility. Previously, Robotic Assisted (RA)-laparoscopic surgery was the procedure of choice when treating stage IV endometriosis. Single Incision Laparoscopic Surgery (SILS) is a new technique, gaining popularity for its versatile use in various surgical procedures. We present a case report of a patient with stage IV appendiceal endometriosis managed with Da Vinci RA-SILS appendectomy and a literature review.
\end{abstract}

Case: The patient was a 34-year-old female Gravida 0, Para 0 with a chief complaint of dysmenorrhea, chronic pelvic pain and cramping. She reported a history of infertility for 18 months. The patient was diagnosed with a right pelvic sidewall nodule with endometriosis, left round ligament endometriosis, left anterior broad ligament endometriosis, posterior cul-de-sac endometriosis, with bladder, uterine, and appendiceal endometriosis. The patient underwent hysteroscopy, chromotubation, and Da Vinci RA-SILS resection of appendiceal endometriosis.

Conclusion: Although rare, endometriotic involvement of appendix can be a symptomatic complication of gastrointestinal involvement and may present as acute appendicitis or even nonspecific abdominal pain, requiring appendectomy. With modern advancements, it is now preferred to perform Da Vinci RA-SILS appendectomy in the setting of endometriosis for both surgical and cosmetic reasons.

Keywords: appendiceal endometriosis, appendix, chromotubation, da vinci SILS appendectomy, da vinci SILS resection of endometriosis, deep-infiltrating endometriosis, dyschezia, dysmenorrhea, endometriosis, endometriosis of appendix, invasive endometriosis, laparoscopy, single-site robotic excision of endometriosis, stage IV endometriosis
Volume 9 Issue 4 - 2018

\author{
Shadi Rezai, ${ }^{1,5}$ Neil D Patel BS, ${ }^{2}$ Alexander \\ C Hughes, ${ }^{2}$ Ninad M Patil, ${ }^{3}$ Elise Bardawil, ${ }^{5}$ \\ Cassandra E Henderson, ${ }^{4}$ Xiaoming Guan ${ }^{5}$ \\ 'Department of Obstetrics and Gynecology, Southern California \\ Kaiser Permanente, USA \\ ${ }^{2}$ St. George's University, School of Medicine, St. George's, \\ Grenada \\ ${ }^{3}$ Department of Pathology \& Immunology, Baylor College of \\ Medicine, USA \\ 4Maternal and Fetal Medicine, Department of Obstetrics and \\ Gynecology, Lincoln Medical and Mental Health Center, USA \\ ${ }^{5}$ Division of Minimally Invasive Gynecologic Surgery, \\ Department of Obstetrics and Gynecology, Baylor College of \\ Medicine, USA
}

\begin{abstract}
Correspondence: Xiaoming Guan MD PhD, Section Chief and Fellowship Director, Division of Minimally Invasive Gynecologic Surgery, Department of Obstetrics and Gynecology, Baylor College of Medicine, 665I Main Street, $10^{\text {th }}$ Floor, Houston, Texas, 77030, USA, Tel (832) 826-7464, Fax (832) 825-9349, Email xiaoming@bcm.edu
\end{abstract}

Received: June 01, 2017 | Published: August 01, 2018

\section{Background}

Endometriosis is defined as functional endometrial tissue located outside of the uterine cavity (ectopic), often involving other structures within the rest of the genital tract or pelvic peritoneum. ${ }^{1}$ The condition primarily affects up to $10 \%$ of reproductive age women. ${ }^{1}$ Several theories have been proposed to explain the pathophysiology of endometriosis; most common ones being retrograde menstrual endometrial implantation or peritoneal lining metaplasia. The symptoms typically experienced by patients with endometriosis stem from endometriotic tissue involving specific structures. Typical symptoms of endometriosis include dysmenorrhea, non-cyclic dyspareunia, irregular or heavy menstrual bleeding, dyschezia, and infertility.

Endometriotic involvement of the gastrointestinal (GI) tract may cause a spectrum of symptoms, ranging from simple to complex. ${ }^{2}$ However, a third of patients may be asymptomatic; and diagnosed incidentally. ${ }^{3}$ Endometriosis is a clinical diagnosis of exclusion, and diagnosis of endometriosis is established through laparoscopic procedures retrieving tissue biopsy. ${ }^{3}$ A definitive diagnosis of endometriosis can only be made by histological evaluation of lesions removed during surgery. ${ }^{4}$ Neither Serum markers nor imaging studies are diagnostic - rather only support the diagnosis of endometriosis. ${ }^{4}$
The histologic appearance consists of endometrial glands and stroma with varying amounts of inflammation and fibrosis - unique to endometriosis. ${ }^{4}$ Histological evaluation is crucial because visual appearance of the lesions during laparoscopy is variable - studies have reported a marked discrepancy between the visual appearance and the histology. ${ }^{4}$ Robotic-assisted (RA) - laparoscopic surgery, has shown promising results with regards to pain relief, laparotomy conversions or complication when treating stage IV endometriosis.

The most common sites for endometriotic involvement are the ovaries $(54.9 \%)$, posterior broad ligament $(35.2 \%)$, anterior cul-desac $(34.6 \%)$, posterior cul-de-sac $(34.0 \%)$ and uterosacral ligament. ${ }^{3}$ Occasionally, endometriotic tissue may be detected within the GI tract, or very rarely, the respiratory tract. ${ }^{3}$ Although endometriotic involvement of the appendix is rare $(0.8 \%)$, it can present with variety of symptoms, or be asymptomatic.,6 Even rarer are severe symptoms of underlying appendiceal endometriosis presenting as acute appendicitis or acute abdomen. ${ }^{7-9}$ Laparoscopic appendectomy is the gold standard treatment for endometrial appendiceal because it allows for thorough surgical exploration of the abdomen, especially in patients with recurrent or unexplained pelvic pain..$^{2,6,10}$

Single Incision Laparoscopic Surgery (SILS) is a new technique, gaining popularity for its versatile use in various surgical procedures. 
Current applications for the procedure include, but are not limited to: tubal ligation, hysterectomy, appendectomy, cholecystectomy, gastrectomy, colectomy, and nephrectomy. ${ }^{11}$ The procedure is gaining traction because of reduced post-operative pain or complications, and cosmetic advantages. ${ }^{11}$ We present a case report of a patient with stage IV appendiceal endometriosis managed with Da Vinci RA-SILS appendectomy and a literature review.

\section{Presentation of the case}

The patient was a 34-year-old female, Gravida 0 Para 0 , with a chief complaint of dysmenorrhea, chronic pelvic pain and cramping, with Mittelschmerz, since menarche at age 13. Her pain was unresponsive to medications and worsening over the past year. She reported regular menses every $24-26$ days. In addition, the patient also reported new-onset dyschezia and dysuria with urinary frequency and urgency. The patient denied dyspareunia however reported post-coital bleeding. She reported a history of infertility and had been trying to conceive for the past 18 months. She did not report any history of hospitalization or surgery.

Physical exam revealed vital signs within normal limits, and BMI of $23.35 \mathrm{~kg} / \mathrm{m}^{2}$. Positive findings included pain on palpation of a bladder nodule and a left vaginal fornix nodule. There were no palpable adnexal masses; rectal exam was within normal limits. A hysterosalpingogram (HSG) one year prior had shown bilateral tubal patency and no uterine filling defects. Her most recent transvaginal ultrasound showed adenomyosis. Pelvic MRI showed a $3.8 \mathrm{~cm}$ complex, septated, low signal intensity, non-enhancing lesion arising from the left adnexa, possibly representing an endometrioma. There are epithelial changes along the bladder and uterosacral ligaments with no evidence of endometriosis (Figure 1).
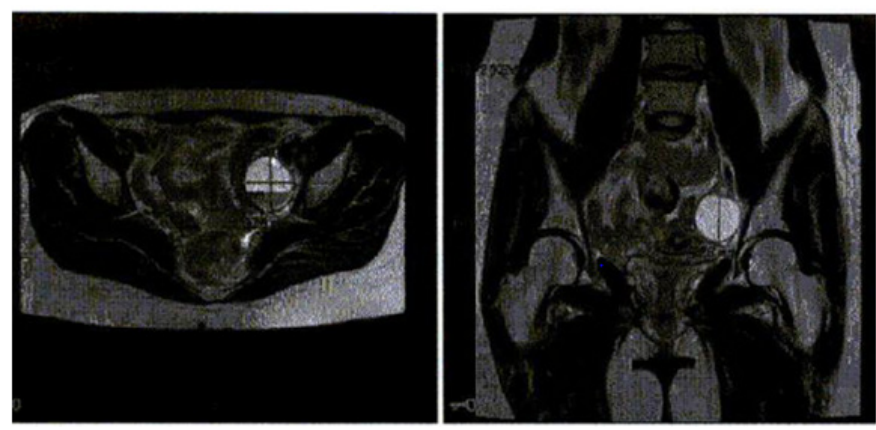

Figure I Pelvic MRI showed a $3.8 \mathrm{~cm}$ complex septated low signal intensity non-enhancing lesion arising from the left adnexa, possible representing and endometrioma. There are epithelial changes along the bladder and uterosacral ligaments with no evidence of endometriosis.

The patient underwent hysteroscopy, chromotubation, and Da Vinci RA-SILS resection of endometriosis. During surgery, patient was noted to have stage IV endometriosis with appendiceal involvement (Figure 2A \& 2B). This was managed surgically by general surgery using Da Vinci RA-SILS appendectomy (Figure 2C). In addition, cystoscopy, and ureteral stent placement and removal were performed by Urology. Intraoperative findings and pathologic specimen evaluation confirmed the diagnosis of right pelvic side wall nodule with endometriosis, left round ligament endometriosis, left anterior broad ligament endometriosis, posterior cul-de-sac endometriosis, with bladder, uterine, and appendix endometriosis.

\section{SILS technique (gynecology/urology)}

Initially, a $15 \mathrm{~mm}$ port access incision was made at the umbilicus entering the peritoneum and docking the robot. Using the monopolar hook and blunt dissection, the left ovary and fallopian tube were freed from the left pelvic sidewall, and a portion of the posterior cul-de-sac peritoneum was removed. Subsequently, endometriosis and nodules involving right and left pelvic sidewall, left round ligament, anterior broad ligament, and anterior uterus were carefully removed. The Urology team carefully dissected off the bladder from the anterior uterine endometriosis nodule. Hemostasis was achieved with the bipolar. The pelvis and abdomen were inspected, and hemostasis was noted throughout. Chromotubation was performed with immediate spill of dye from the right fallopian tube and delayed spill of dye from the left fallopian tube. An Interceed absorbable adhesion barrier was placed over the area of dissection along the left pelvic sidewall and the anterior surface of the uterus and bladder.

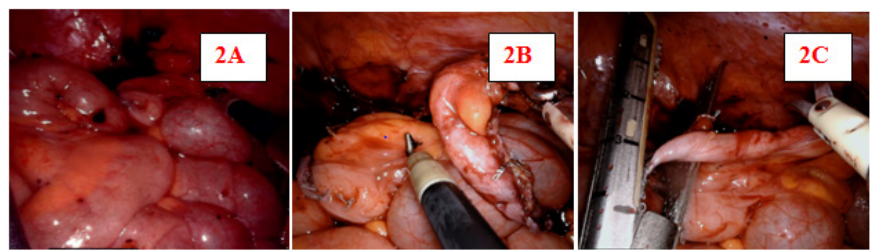

Figure 2 Intraoperative Images: 2A: Multiple endometriotic implants on the bowel; 2B: Appendix/Bowel; 2C: The appendix stapled off with a tan reload stapler and the mesentery with a gray reload stapler.

\section{RA-SILS appendectomy technique}

RA-SILS access was established via the $15 \mathrm{~mm}$ umbilical incision. The appendix was visualized with endometriosis affecting primarily the retroperitoneal tip. The affected area was incised laterally to mobilize the cecum, and the appendix was dissected out until the base was identified. At this point the $12 \mathrm{~mm}$ port was inserted on the left side and the appendix was stapled off with a tan reload stapler and the mesentery with a grey reload stapler. The appendix, including the surrounding peritoneum with endometriosis was excised, placed in a bag, and removed via the single site port site. Multiple specimens were collected and sent to pathology. Pathologic examination confirmed the presence of endometriosis in the right pelvic side wall nodule, left round ligament, left anterior broad ligament, bladder and uterine serosa, and outer appendiceal wall (Figure 3). At postoperative 3-week follow-up, the patient reported normal bowel movements and only mild left pelvic pain.
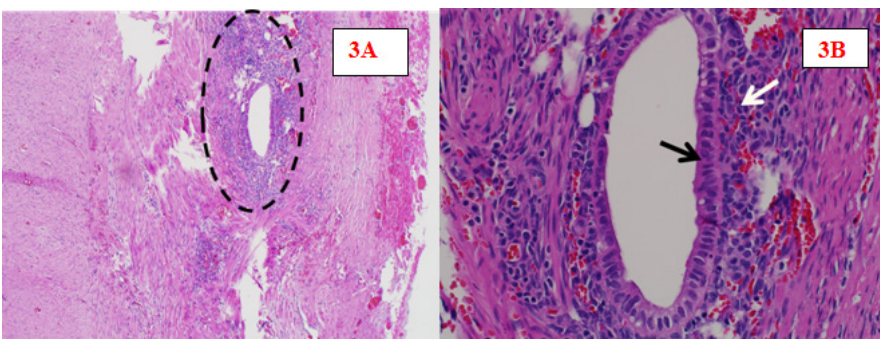

Figure 3 Pathology Images: 3A: At low power, endometriosis (encircled) is noted in the outer appendiceal muscularis (H\&E); 3B: On higher power, an endometrial-type gland (black arrow) surrounded by endometrial-type stroma (white arrow) is seen within smooth muscle (H\&E). 


\section{Discussions}

The signs and symptoms of endometriosis can be nonspecific, or it may even be asymptomatic. However, endometriosis remains a leading cause of chronic pelvic pain in reproductive age women. Although rare, endometriosis can involve the appendix causing pain and distress; or be found incidentally. Similar to acute appendicitis, the laparoscopic surgical approach is considered to be the gold standard. As cosmetic outcomes have become more important in surgery, minimally invasive or SILS procedures are being used more often. Taking a traditional approach and adding a modern twist, SILS appendectomy is now an acceptable procedure to surgically treat symptomatic and asymptomatic appendix-focused conditions. The main benefits come from ease of access within the abdominal cavity, with gentle tissue handling and manipulation. In addition, SILS allows for proper tissue dissection and the use of the Endoloop as an alternative to the larger trocar for stapler use. ${ }^{12}$

Prior to SILS, Double-incision laparoscopic appendectomy (DILA) was introduced as a means of further minimizing the invasiveness of traditional laparoscopic surgery, by decreasing then number of incisions. ${ }^{13}$ A randomized control trial has demonstrated that DILA can be performed with equivalent operative outcomes with superior cosmetic outcomes. ${ }^{13}$ Successful operations using DILA urged the push for SILS, as stated previously, primarily for cosmetic reasons. ${ }^{11}$ In direct comparison to traditional laparoscopic appendectomy, SILS appendectomy can be conducted with comparable operative times, lengths of stay, and procedural complications, and with superior cosmetic outcomes - the main downside noted being the cost. ${ }^{14,15}$ As reported in this case, the use of SILS can be easily manipulated using conventional laparoscopic instruments. A previous study outlined SILS appendectomy utilizing three trocars (two $10 \mathrm{~mm}$ and one $5 \mathrm{~mm}$ ), using conventional and optical laparoscopic tweezers $\left(10 \mathrm{~mm}, 30^{\circ}\right)$; while using titanium LT 400 clips to ligate the base and pedicle of the appendix. $^{11}$

As described earlier, the SILS procedure can be applied to appendectomy in female patients suffering from endometriosis. A previous study performed to evaluate deeply located endometriosis showed significant involvement of the appendix. Out of the patients evaluated, nearly $50 \%$ have deep involvement by endometriosis, while $32 \%$ showed appendiceal involvement. ${ }^{16}$ Although these patients were asymptomatic, the level of infiltration was indicative for surgical intervention. ${ }^{16}$ It has been suggested that prophylactic concurrent appendectomy be performed as part of complete endometriosis excision. ${ }^{16}$ Unusually, other intra-abdominal pathology can be mimicked by pain from appendiceal endometriosis. ${ }^{17}$ Isolated, deeply located appendiceal involvement may be missed by diagnostic laparoscopy; and can present with no external diagnostic features, therefore routine appendectomy has also been advocated in women with recurrent abdominal pain..$^{17-20}$

\section{Conclusion}

In the majority of patients, endometriosis presents very classically - dysmenorrhea, non-cyclic dyspareunia, irregular or heavy menstrual bleeding, dyschezia, and infertility. Rare presentations can be due to endometriotic involvement of the gastrointestinal tract, presenting an array of symptoms, but may also be asymptomatic. Although rare, endometriotic involvement of appendix can be a symptomatic complication of gastrointestinal involvement and may present as acute appendicitis or even nonspecific abdominal pain, requiring appendectomy. Traditionally, both endometriosis and appendicitis have been evaluated and treated laparoscopically. It has been proposed, that indicated endometrial appendix removal be performed by appropriately trained gynecologists. ${ }^{21}$ With modern advancements, it is now preferable to perform Da Vinci RA-SILS appendectomy in the setting of endometriosis for both surgical and cosmetic reasons.

\section{Acknowledgments}

Dr. Xiaoming Guan is a speaker for Applied Medical, Rancho Santa Margarita, and California.

\section{Conflicts of interest}

Authors did not report any potential conflicts of interests.

\section{References}

1. Murphy SJ, Kaur A, Wullschleger ME. Endometrial decidualization: a rare cause of acute appendicitis during pregnancy. J Surg Case Rep. 2016;2016(4)

2. Yoon J, Lee YS, Chang HS, et al. Endometriosis of the appendix. Ann Surg Treat Res. 2014;87(3):144-7.

3. Saleem A, Navarro P, Munson JL, et al. Endometriosis of the appendix: Report of three cases. Int J Surg Case Rep. 2011;2(2):16-9.

4. ACOG Practice bulletin no. 114: management of endometriosis. Obstet Gynecol. 2010;116(1):223-36.

5. Brudie LA, Gaia G, Ahmad S, et al. Peri-operative outcomes of patients with stage IV endometriosis undergoing robotic-assisted laparoscopic surgery. J Robot Surg. 2012 Dec;6(4):317-22.

6. Paramythiotis D, Stavrou G, Panidis S, et al. Concurrent appendiceal and umbilical endometriosis: a case report and review of the literature. $J \mathrm{Med}$ Case Rep. 2014;8:258.

7. Ben Maamer A, Zaafouri H, Haoues N, et al. Laparoscopic appendectomy for appendiceal endometriosis presenting as acute appendicitis. Tunis Med. 2013;91(5):369-70.

8. Emre A, Akbulut S, Yilmaz M, et al. An unusual cause of acute appendicitis: Appendiceal endometriosis. Int J Surg Case Rep. 2013;4(1):54-7.

9. Salati SA, Raza AA. Endometriosis: a rare cause of appendicitis. $J$ Coll Physicians Surg Pak. 2011;21(5):304-5.

10. Laskou S, Papavramidis TS, Cheva A, et al. Acute appendicitis caused by endometriosis: a case report. J Med Case Rep. 2011;5:144.

11. Pinheiro RN, Sousa RC, Castro FM, et al. Single-incision videolaparoscopic appendectomy with conventional videolaparoscopy equipment. Arq Bras Cir Dig. 2014;27(1):34-7.

12. Kliethermes C, Blazek K, Guan X. Single-Incision Robotic Appendectomy, Video Posters - Endometriosis, Video Number 430. J Minim Invasive Gynecol. 2016;23(7):S143.

13. Pattanshetti VM, Krishna KL. Conventional laparoscopic appendectomy versus double-incision, three-port laparoscopic appendectomy: A 1-year randomized controlled trial. Asian J Endosc Surg. 2018.

14. Buckley FP, Vassaur H, Monsivais S, et al. Single-incision laparoscopic appendectomy versus traditional three-port laparoscopic appendectomy: an analysis of outcomes at a single institution. Surg Endosc. 2014;28(2):62630 .

15. Villalobos Mori R, Escoll Rufino J, Herrerías González F, et al. Prospective, randomized comparative study between single-port laparoscopic appendectomy and conventional laparoscopic appendectomy. Cir Esp. 2014;92(7):472-7. 
16. Moulder JK, Siedhoff MT, Melvin KL, et al. Risk of appendiceal endometriosis among women with deep-infiltrating endometriosis. Int $J$ Gynaecol Obstet. 2017;139(2):149-154.

17. Shen AY, Stanes A. Isolated Appendiceal Endometriosis. J Obstet Gynaecol Can. 2016;38(10):979-981.

18. Fatehchehr S, Macik P, Sinervo K. Small Bowel Segmental Resection and Reanastomosis with Appendectomy for Invasive Endometriosis. J Minim Invasive Gynecol. 2015;22(6S):S138-S139.

19. Ribeiro DM, Ribeiro GP, Santos TP, et al. Incidental Appendectomy in the Surgical Treatment of Deep Endometriosis Infiltrating the Bowel: Anatomo-pathological Findings in a Series of 109 Patients. J Minim Invasive Gynecol. 2015;22(6S):S30-S31.
20. Shavell VI, Mahdi HM, Awonuga AO, et al. Appendectomy in the gynecological setting: intraoperative findings and corresponding histopathology. Gynecol Obstet Invest. 2011;71(3):189-92.

21. Jocko JA, Shenassa H, Singh SS. The role of appendectomy in gynecologic surgery: a Canadian retrospective case series. J Obstet Gynaecol Can. 2013;35(1):44-8. 